\title{
EDITORIAL
}

\section{Journey of the Journal}

The Journal of National Science Foundation (JNSF) of Sri Lanka is a multidisciplinary journal and publishes original articles with an emphasis on the latest research findings. The journal aspires to bring eminent research information across the globe into light and aims to build an efficient platform for researchers all over the world.

To fulfill the above vision, the journal is released four times a year, in March, June, September and December, and it contains Research Articles, Reviews, Research Communications and Correspondences. The journal is available both in print as well as online. From March 2008, the Journal is indexed in Science Citation Index Expanded, Journal Citation Reports/Science Edition, BIOSIS Previews, Zoological Record, Biological Abstracts and later in Ulrich's, Agricola, Scopus and EBSCOhost.

As a multidisciplinary journal, submissions from different disciplines vary in each year. For example, when considering the percentage of submissions by discipline in 2017, the highest percentage (20\%) is from the Engineering field. The second highest percentage $(10.4 \%)$ is from Information Technology. The percentage of submissions in the other fields are, Mathematics (9.2\%), Statistics (9.2\%), Agriculture (8.8\%), Chemistry (6.4\%), Zoology (5.2\%), Botany (4.8\%), Microbiology (4.4\%), Medicine (4\%), and the rest of the fields are less than $4 \%$.

Although the journal is indexed in the Science Citation Index Expanded from March 2008, the first impact factor of 0.134 was assigned in 2010. Manuscript submission to the journal from foreign authors gradually increased from 2011 onwards. In 2008, 91\% of the submissions were from local authors. However, the percentage of submissions by foreign authors increased from $7 \%$ to $70 \%$ during the period from 2008 to 2018 . The total number of submissions in 2008 was 68. In 2018, the journal has received 342 manuscripts. Out of these, 241 were foreign submissions. The number of submissions from local authors was 89 and 12 articles were authored by both local and foreign authors during this period.

All submitted manuscripts of the journal undergo an initial screening by the journal's editorial staff to ensure that the content of manuscripts is adequate for a full paper having sufficient scientific merit, manuscripts adhere to all instructions in the Author Guidelines, and that they are well written and do not have a substantial similarity to already published work. Based on the statistics related to the JNSF from 2008-2018, manuscript rejection rate at the initial screening was high, mainly due to submissions with low scientific merit, and poor presentation.

The JNSF practices a rigorous double-blind review, wherein the identities of both authors and reviewers are not revealed during the peer review process. The time taken to process an article from submission to acceptance or rejection is mainly based on the review process. The journal maintains a reviewer pool to handle the manuscripts efficiently. Currently, 4588 local reviewers and 152 foreign reviewers are in this pool. The average time taken for the different stages of the review process has been analyzed based on the statistics for the period 2008-2017. The average time taken from submission to publication is $13-17$ months. The time taken from receipt of manuscript to acceptance is on an average 5 to 11 months, and 8 - 9 months from acceptance to publications. The average time taken for good quality papers is short. If the manuscript has to go through several revisions starting from a major revision, the final decision for publication may be delayed. Identifying reviewers in some specific areas and delayed response from reviewers and authors are two other reasons for the delay in making the final decision regarding a manuscript.

Average acceptance rate of manuscripts from different disciplines varied from $17 \%$ to $47 \%$ over the period from 2008 to 2017. Manuscripts of Molecular Biology had the 
highest percentage (46\%) of acceptance. The percentage of acceptance of manuscripts in other disciplines varied from $33 \%$ to $19 \%$; Physics \& Nuclear Science (33\%), Chemistry (33\%), Statistics (31\%), Botany (31\%), Mathematics (27\%), Geology (27\%), Zoology (26\%), Microbiology (24\%), Electronics \& Computer Science (23\%), Engineering (20\%), and Medicine (19\%).
It is observed that most of the local researchers still submit their research findings with high scientific merit to foreign journals. Attracting such manuscripts having a substantive contribution to new knowledge and improving the impact factor are among the challenges faced by the JNSF.

Pushpa Wijekoon 